\title{
Effects of Shared Gaze Parameters on Visual Target Identification Task Performance in Augmented Reality
}

\author{
Nahal Norouzi* \\ University of Central Florida \\ nahal.norouzi@knights.ucf.edu \\ Ryan Schubert \\ University of Central Florida \\ rschuber@ist.ucf.edu
}

\author{
Austin Erickson* \\ University of Central Florida \\ ericksona@knights.ucf.edu \\ Joseph J. LaViola Jr. \\ University of Central Florida \\ jjl@eecs.ucf.edu \\ Gregory F. Welch \\ University of Central Florida \\ welch@ucf.edu
}

\author{
Kangsoo Kim \\ University of Central Florida \\ kangsoo.kim@ucf.edu \\ Gerd Bruder \\ University of Central Florida \\ bruder@ucf.edu
}

\begin{abstract}
Augmented reality (AR) technologies provide a shared platform for users to collaborate in a physical context involving both real and virtual content. To enhance the quality of interaction between AR users, researchers have proposed augmenting users' interpersonal space with embodied cues such as their gaze direction. While beneficial in achieving improved interpersonal spatial communication, such shared gaze environments suffer from multiple types of errors related to eye tracking and networking, that can reduce objective performance and subjective experience.

In this paper, we conducted a human-subject study to understand the impact of accuracy, precision, latency, and dropout based errors on users' performance when using shared gaze cues to identify a target among a crowd of people. We simulated varying amounts of errors and the target distances and measured participants' objective performance through their response time and error rate, and their subjective experience and cognitive load through questionnaires. We found some significant differences suggesting that the simulated error levels had stronger effects on participants' performance than target distance with accuracy and latency having a high impact on participants' error rate. We also observed that participants assessed their own performance as lower than it objectively was, and we discuss implications for practical shared gaze applications.
\end{abstract}

\section{CCS CONCEPTS}

- Human-centered computing $\rightarrow$ Empirical studies in visualization; • Computing methodologies $\rightarrow$ Mixed / augmented reality.

*Both authors contributed equally to this research.

Permission to make digital or hard copies of all or part of this work for personal or classroom use is granted without fee provided that copies are not made or distributed for profit or commercial advantage and that copies bear this notice and the full citation on the first page. Copyrights for components of this work owned by others than ACM must be honored. Abstracting with credit is permitted. To copy otherwise, or republish, to post on servers or to redistribute to lists, requires prior specific permission and/or a fee. Request permissions from permissions@acm.org.

SUI '19, October 19-20, 2019, New Orleans, LA, USA

(c) 2019 Association for Computing Machinery.

ACM ISBN 978-1-4503-6975-6/19/10 . \$ \$15.00

https://doi.org/10.1145/3357251.3357587

\section{KEYWORDS}

Augmented Reality; Shared Gaze; Eye Tracking; Target Identification

ACM Reference Format:

Nahal Norouzi, Austin Erickson, Kangsoo Kim, Ryan Schubert, Joseph J. LaViola Jr., Gerd Bruder, and Gregory F. Welch. 2019. Effects of Shared Gaze Parameters on Visual Target Identification Task Performance in Augmented Reality. In Symposium on Spatial User Interaction (SUI '19), October 19-20, 2019, New Orleans, LA, USA. ACM, New York, NY, USA, 11 pages. https: //doi.org/10.1145/3357251.3357587

\section{INTRODUCTION}

Over the last several years, great strides have been made to improve sensor and display technologies in the fields of augmented reality (AR) and virtual reality (VR) [16]. These advances, such as with respect to head-mounted displays (HMDs) and eye trackers, have provided new opportunities for applications in fields such as training, simulation, therapy, and medicine. For many of these, collaboration between multiple users is an important aspect of the experience. In real life, people use both verbal and nonverbal cues to communicate information to the person they are interacting with.

In order to understand and improve collaborative experiences using AR/VR technologies, researchers have evaluated the impact of different embodied and behavioral cues on users' efforts and performance [18, 26, 27]. Researchers have also investigated how certain embodied cues could be augmented to improve their efficiency in interpersonal communication. A prime example of such augmented cues are shared gaze environments [26]. Eye gaze is an important cue for spatial interaction and collaboration among humans as it lets us know what another person is looking at, which is often in line with their focus of attention [20]. Gaze cues further inform us about a person's mental processes, eye contact, and gaze avoidance. foint gaze occurs when multiple people are looking at the same object when it is the focus of discussion [17].

Advances in AR requires a better understanding of its interaction space [32], such as AR technologies' potential to augment such eye gaze cues in interpersonal communication, e.g., by providing a gaze ray from the user's head to the object in the environment that they are looking at. Different methods have been proposed to share gaze 
rays, cones, or focus points between users [18, 27,33]. Some of these approaches require information about the geometry of the physical environment, while the most basic and generalizable approaches are mainly based on rays that indicate the direction of gaze but do not terminate at any object.

All shared gaze environments have to track users' gaze direction with an eye tracker, e.g., integrated into an HMD, and that they have to transmit that information others for subsequent rendering in their AR view, e.g., via a wireless network. The quality of shared information and the errors involved in this process are highly important to quantify and understand for practitioners in shared spaces [7, 28, 33].

In this paper, we present a human-subject study aimed at understanding the importance and influence of four types of errors in AR shared gaze environments on users' performance and perception. We focused on accuracy and precision related to the eye tracker as well as latency and dropout related to the network. We created a scenario where a participant had to collaborate with a simulated partner to identify a target among a crowd of humans. Inspired by related work [18, 27, 33], we augmented the participant's view with a gaze ray that indicated which person their simulated partner was looking at. We measured participants' performance using response time and error rate. Our findings suggest that participants performed surprisingly well, better than they believed themselves when we asked them to judge their performance, even when the simulated error levels were high, with accuracy and latency having the highest impact on participants' error rates.

With this work, we aim to contribute to the research community by providing answers to the research questions below:

- RQ1 How do different types of error affect users' collaborative performance in AR shared gaze environments?

- RQ2 What are the thresholds for the amounts of error introduced without affecting users' performance?

- RQ3 How different are users' subjective and objective assessment of the experience and what is their subjective perception of an acceptable amount of error for the task at hand?

This paper is structured as follows. Section 2 discusses related work. Section 3 describes the experiment. Section 4 describes our results and Section 5 discusses our findings. Section 6 concludes the paper and discusses future opportunities for research.

\section{RELATED WORK}

In this section, we present related work on collaborative shared spaces, shared gaze cues, and errors impacting user experience and performance.

\subsection{Sharing Gaze in AR/VR}

In an early work by Kiyokawa et al., a mixed-space collaborative platform was introduced that included an awareness enhancing technique to improve the quality of collaboration between the two users [18]. This was achieved by visualizing a gaze ray initialized from between the user's eyes, finding that participants rated their task to be easier with the gaze ray when one participant had to guide the other in finding stationary targets. Similarly, Bauer et al. investigated the effects of a "reality augmenting telepointer" used for expert-worker scenarios for mobile workers. Using their system, the expert's pointer was displayed to the user [2]. They found that with the inclusion of the pointer, similar speech behaviors were observed as in face-to-face conversations. Piumsomboon et al. introduced a system called COVAR that could facilitate collaboration between AR and VR users and is able to share their head frustum related to their field of view, head-ray, gaze-ray, and hand gestures of users with each other to improve the collaborative experience [27]. Their results emphasize the positive impact of these cues on aspects such as performance and subjective preference. Brennan et al. compared different combinations of gaze and voice cues where remote users took part in a search task finding that sharing gaze information alone resulted in faster search times than gaze and voice conditions [3]. In a helper-worker scenario, Gupta et al. looked at effects of sharing the worker's gaze with the helper and the helper's pointer with the worker. Their results indicate positive impacts of having both cues on performance and quality of the experience [11]. With such findings emphasizing the benefits of shared gaze, Zhang et al. investigated the impact of the methods used to visualize the gaze (i.e., highlight, spotlight, trajectory, and cursor) on users' performance and cognitive load [33]. Their findings suggest that users perceived the highlight and spotlight mode as less distracting compared to other modes such as cursor and trajectory. Although past research have had valuable contributions in understanding the influence of sharing gaze in AR/VR for collaborative purposes, to our knowledge, the influence of different types of errors inherent to shared gaze experiences have not been studied. Knowing these influences exist in single user experiences, a better understanding is required for shared experiences.

\subsection{Gaze Tracking Performance}

As the quality of the data reported by eye trackers is dependent on various factors, understanding the causes and effects of these issues on user performance is important for practical applications. Holmqvist et al. discussed basic examples of different types of errors in eye tracking environments that are caused by limitations of current-state eye trackers (e.g., accuracy and precision), and how these can cause misinterpretations in different measures such as dwell time [13]. They discussed factors that can affect the quality of the data such as the experimental task and eye tracking algorithms. Other researchers also provided explanations for sources of eye tracking errors such as variations in pupil size [6], eye color as well as calibration instructions and methods [23].

Some researchers proposed methods to compensate for these errors. For instance, Cerrolaza et al. proposed calibration techniques to compensate for the impact of user movement on the devices' accuracy [5]. For less expensive commercial off-the-shelf (COTS) eye trackers, Ooms et al. proposed steps to improve their data quality [24]. Hornoff et al. proposed using the disparity between the true position of implicit fixation points and the reported value from the eye tracker as a way to measure the robustness of the reported data [14]. Barz et al. proposed a computational approach that would model and predict gaze estimation errors in real time and could be used in applications to identify high error regions during user interaction and modify the elements such as increasing the size of the objects [1]. While varying factors such as lighting and eye 
tracker type, Feit et al. found large differences in eye tracking data quality and proposed new design choices such as target placement and size adjustments to compensate for these variabilities [8].

With most of past work's focus on assessing the gaze tracking errors, identifying contributing factors and solutions reducing their impact, further investigation of these errors in more dynamic shared AR/VR setups can be beneficial.

\subsection{Network Sharing Performance}

Network performance in terms of transmission latency and dropout is an important factor that shapes user performance in shared AR/VR experiences. A large body of literature showed that latency has a negative effect on user performance, but most of that research focused on tracker or rendering latency between a person's own physical movements and the computer-generated feedback [7, 15, $19,21,25,28]$. In contrast, effects of network latency and dropout in shared AR/VR environments have less immediate cause-effect relationships.

We are not aware of previous research investigating latency and dropout in shared gaze environments, but related work in AR/VR and general communication focused on aspects of collaborative environments [10, 29]. Recently, Toothman and Neff investigated different network errors in an embodied multi-user VR setup, including latency and dropout and their effects on social presence [30]. Their results showed practical thresholds such as that a latency of $300 \mathrm{~ms}$ and dropout with freezing frames for $100-350 \mathrm{~ms}$ for $67 \%$ of the time had a negative impact on users' experience and performance. dropout (or frame dropping) has been further researched by Pavlovych et al., who identified a threshold of $10 \%$ frame drops, after which it had a negative effect on participants' tracking task performance on a computer [25]. Geelhoed et al. showed that the conversation flow in a telepresence system was reduced by added network latency, recommending a limit of 100-600 ms for round trip time latency, but they also found that basic conversations without time sensitive tasks were not that affected by latency and could go up to $2000 \mathrm{~ms}$ of latency [10]. Other research showed that network latency might further lead to misinterpretations of users' dispositions during interactions [29].

Further investigation of different error types for collaborative purposes can provide a better understanding of their implications on users' collaborative performance and experience.

\section{EXPERIMENT}

In this section, we present the experiment that we conducted to assess the impact of different types of errors that are inherent to collaborative shared gaze environments in AR.

\subsection{Participants}

We recruited 21 participants (7 female, 14 male, age 19-36, average 23.28) from the graduate and undergraduate population of our university. The protocol for our experiment was approved by the institutional review board (IRB) of our university. All participants indicated normal hearing and normal/corrected vision. Before the experiment, we asked our participants to use a 7-point Likert scale ( $1=$ novice/unfamiliar, 7 = expert/familiar $)$ to rate their familiarity with AR (average 4.7), VR (average 5.19), virtual humans (average 3.9), and overall computer expertise (average 5.52).

\subsection{Material}

We conducted the experiment in an open $4.6 \mathrm{~m} \times 10.4 \mathrm{~m}$ space in our laboratory. We used two computers with Intel Xeon $2.4 \mathrm{GHz}$ processors comprising 16 cores, $32 \mathrm{~GB}$ of main memory and two Nvidia Geforce GTX 980 Ti graphics cards for the stimulus control and for participants to answer questionnaires. We used the Unity graphics engine version 2018.2.11f1 for rendering, and a Microsoft HoloLens for the presentation of the visual stimulus.

3.2.1 Shared Gaze Stimuli. To provide repeatable controlled shared gaze stimuli, we decided to use a simulated virtual human partner in this study. Therefore, we placed a 3D virtual human character (see Figure 1) at a distance of one meter on the left side to the participants, which was visible to them on the HoloLens. During the experiment, the simulated partner stood with an idle animation, facing in the same forward direction as the participants. A 20-meter gaze ray was presented in AR that originated in the partner's eye location and went forward into the environment. The gaze ray was programmed to be rendered on top of the real or virtual entities in the environment to be more in line with practical shared gaze setups and to not give away or misrepresent a target through depth cues resulting from an intersection with a target at any moment. We would like to point out that such gaze rays are mainly used when one does not have access to a high-precision real-time reconstruction of the geometry of a physical environment, as discussed in Section 1.

We tested different gaze simulation algorithms but noticed that these were largely not able to create realistic gaze behavior in AR. In order to create a natural gaze behavior for the simulated partner, we recorded the eye behavior of one of the experimenters looking at a stationary target located at a distance of one meter away. We used the Pupil Labs ${ }^{1}$ software to capture the recording and assess its accuracy $(0.55 \mathrm{deg})$ and precision $(0.08 \mathrm{deg})$. This recorded data was then analyzed to find the average gaze position observed (with the accuracy error), and was then normalized around this position to yield data with no accuracy error.

This recorded gaze data was played back in Unity and oriented to simulate saccades and smooth pursuit movements to follow target points on the moving target humans in the environment. Each of them had three points of interest, one on their head, one on their chest, and one near their waist (see Figure 1).

The script that controlled the gaze behavior would target one of these points at random every $750 \mathrm{~ms}$, with a fifty-percent probability of choosing the head as the target and a twenty five percent chance of choosing either of the other two points. This behavior made the virtual partner's gaze seem as though it was identifying the target human by recognizing their face, while scanning the target for concealed weapons. The simulated gaze followed the targets when they were moving.

\footnotetext{
${ }^{1}$ https://pupil-labs.com/
} 


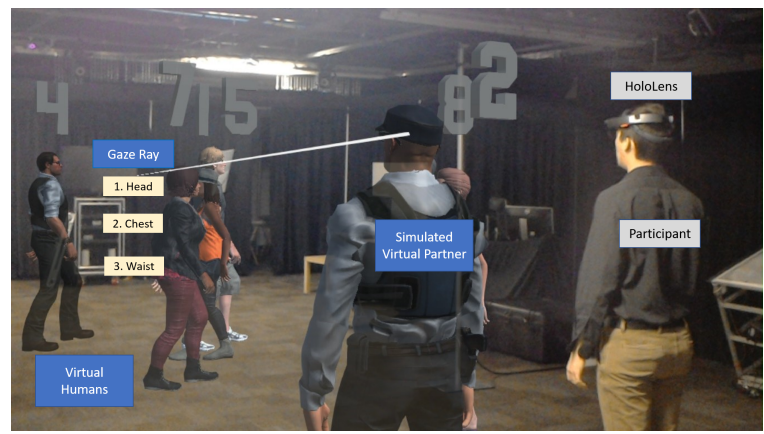

Figure 1: Annotated screenshot showing a participant wearing a HoloLens with the simulated virtual partner on their left side, looking at a target in a crowd of virtual humans. The virtual target humans are differentiated by the floating numbers above their head.

3.2.2 Gaze Target Crowd. Our setup consisted of nine simulated virtual human targets shown in Figure 1. The 3D models and animations were acquired through the Unity Asset Store ${ }^{2}$ or Mixamo ${ }^{3}$ The virtual humans ( 4 female, 5 male) were placed 0.7 meters apart from each other in depth. Walking animations were added to each model so they could pace back and forth between two predefined points on the left and right sides from the participants with a total distance of 6 meters. Each virtual human was presented with a floating number over their head to make it easier for the participants when reporting the gaze target. The walking speed for the virtual humans was chosen from the range $0.8 \pm 0.2 \mathrm{~m} / \mathrm{s}$, which is close to average human walking speed [9]. The order of the virtual humans in depth and their walking speed were randomized between trials in the study.

3.2.3 Gaze Error Implementation. As discussed in Section 1, we considered four different types of error that are common to eye trackers and shared gaze AR experiences. Figure 2 illustrates each error type in comparison to a no-error example. Below, we describe each error, its possible source and how it was implemented.

Accuracy: Persistent angular offset between the true eye gaze direction and the direction of the drawn gaze ray. To implement this error, the gaze ray for no accuracy error was calculated based on the simulated virtual partner's gaze direction and the recorded gaze data. This ray was then rotated towards the rightward horizontal axis of the target by a variable number between 0 and 5 degrees at 1 degree intervals to achieve an accuracy offset along the horizontal axis. It is important to note that while a physical eye tracking system would introduce errors in both the $x$ and $y$ directions, we opted to simulate the most extreme type of accuracy error that could occur for the study scenario, which was a horizontal shift away from the target. Due to the nature of our study scenario, a vertical offset would still appear on the target's body or slightly above the target's head, which would make the target easier to identify than if the shift had occurred in the horizontal direction alone. Additionally, a combination of these two directions would

\footnotetext{
${ }^{2}$ https://assetstore.unity.com/

${ }^{3}$ https://www.mixamo.com/\#/
}

only limit the horizontal offset away from the target, and would also result in a target that is easier to identify than if the shift had occurred in solely the horizontal direction.

Precision: Dynamic angular differences between an eye tracker's reported eye gaze direction and the true direction to the gaze target. To implement this error, we calculated the gaze ray position based on the simulated virtual partner's gaze direction, then offset this position by an amount based on the recorded gaze data which was multiplied by a variable scale factor between 0 and 2.5 degrees at 0.5 degree intervals. This calculation would yield a gaze behavior that was centered on the target with increased variations around the target point as the scale factor increased.

Latency: End-to-end delay in the presentation of the gaze ray from the simulated partner's eyes. Here, our focus is on the latency introduced by the complex setup of a collaborative AR shared gaze system, which includes latency from the eye tracker, a wireless network, a rendering system, and a display. To realize this error, we computed the position of the target virtual human at a simulated temporal offset up to $1000 \mathrm{~ms}$ into the past. This past position was then set as the target for the simulated gaze ray. This was achieved by creating a dictionary that paired vector positions with timestamps for each virtual human in the scene. This dictionary could be searched to find a virtual human's past position based on the time difference between the current time and the amount of latency in milliseconds that was simulated.

Dropout: Here we define dropout as the probability of dropped or lost frames due to networking or eye tracking issues (e.g., eye tracker not being able to detect the pupil). To implement this error, for every gaze ray that was rendered we measured the chance of the next ray being dropped based on predefined values that are introduced in Section 3.3.1. As an example, if the predefined value was set to $90 \%$, then there was a $90 \%$ chance that the next frame was dropped resulting in an inconsistent gaze ray.

To choose ecologically valid error ranges, we looked at the literature described in Section 2 and included the nominal performance reported by manufacturers for commercial head-worn eye trackers such as from Tobii ${ }^{4}$ and Pupil Labs. To make sure that participants were able to perceive at least the maxima of all types of errors, we chose the maxima of our error ranges as slightly larger than the range of values reported in the mentioned sources.

\subsection{Methods}

3.3.1 Study Design. We chose a $4 \times 6 \times 3$ within-subjects design for our experiment. This choice was made to account for the impact of individual differences on task performance. Our independent variables were:

- Error Type and Error Level ( $4 \times 6$ factors):

- Accuracy: 6 levels of accuracy error were introduced to the gaze ray from $0 \mathrm{deg}$ to $5 \mathrm{deg}$ with increments of $1 \mathrm{deg}$.

- Precision: 6 levels of precision error were introduced to the gaze ray from $0 \mathrm{deg}$ to $2.5 \mathrm{deg}$ with increments of $0.5 \mathrm{deg}$.

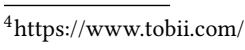



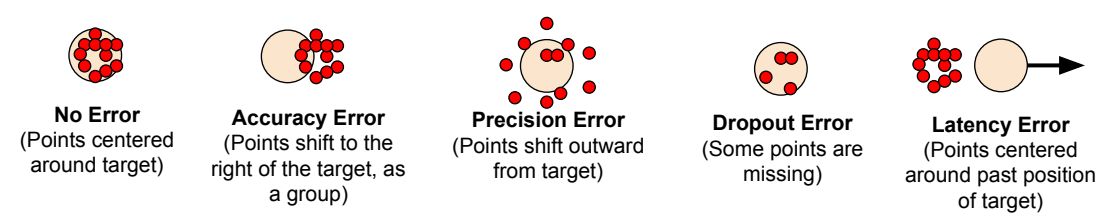

Figure 2: Illustration of the different error types simulated in the shared gaze interface in comparison to a no-error example.

- Latency: 6 levels of latency error were introduced to the gaze ray from $0 \mathrm{~ms}$ to $1000 \mathrm{~ms}$ with increments of $200 \mathrm{~ms}$.

- Dropout: 6 levels of dropout error were introduced to the gaze ray starting at $0 \%$ and going from $10 \%$ to $90 \%$ with increments of $20 \%$.

- Target Distance (3 factors):

- Close: The target was pacing back and forth at a distance of 3 meters.

- Medium: The target was pacing back and forth at a distance of 5.1 meters.

- Far: The target was pacing back and forth at a distance of 7.2 meters.

These combinations of independent variables resulted in a total of 72 trials. For the experiment, we randomized the participants' exposure to each error type resulting in four combinations, and within each error type, we randomized their exposure to the error levels for different target distances, resulting in 18 combinations.

3.3.2 Procedure. After giving their informed consent, participants were asked to take a seat and answer a pre-questionnaire to rate their familiarity with AR, VR, computers, and virtual humans and to assess their hearing and vision. After this, they were asked to read a document about their task in the experiment and then the experimenter reviewed the written document with them.

Participants were guided to stand on the white cross marked on the floor a meter apart from the simulated AR collaboration partner and don the HoloLens. They were informed that they could move their head freely but that they should remain standing on that spot. Participants were asked to use the information from the partner's gaze ray to identify a potential suspect among the gaze target crowd in front of them.

Two distinct beep sounds were used to mark the start and end of each trial and participants were told that they should say their answers out loud by naming the number on top of the identified target human in the crowd. Participants were informed that they have a maximum of 60 seconds to make a decision for each trial but that they have to answer as quickly and confidently as possible. Upon reaching the 55th second in each trial, the virtual background turned red to indicate that the end of the trial was close and participants were asked to indicate their best guess.

After going over the procedure, participants took part in five practice trials. We then asked them if they think they need more practice. If their answer was yes, they took part in five more practice trials. Among our participants, only one asked for the extra five practice trials and we checked the response times for the 72 main trials for that participant to ensure that the extra five practice trials had not given them an advantage over the others.

After each block of 18 trials (i.e., one error type with six error levels and three target distances) they were asked to doff the HoloLens and answer a post-questionnaire. For each participant, this process was performed four times in randomized order within and between the blocks. After finishing the last block, participants were asked to answer further questionnaires. Then, they took part in a short debriefing session to discuss their experience. They received monetary compensation for their participation in the study.

3.3.3 Measures. In this section, we present the objective and subjective measures used to assess participants' performance and perception of each error type.

Objective Measures. As participants were asked to identify the potential gaze target as quickly and confidently as they can, we used these criteria to separately assess their performance in the form of response time and error rate. We would like to point out that although different models for speed-accuracy trade-offs have been introduced in the human-computer interaction literature, we are not aware of any validated model that could be applied to our specific stimulus-response case, such that we had to treat these measures separately:

- Response Time: We recorded the amount of time taken for each participant to indicate a gaze target for each trial.

- Error Rate: We recorded if participants identified the correct gaze target for each trial.

Subjective Measures. We measured the subjective perception of our participants about the various error types and levels, and how their performance and general experience was impacted. The subjective measures used were:

- Subjective Performance: For each block associated with a certain error type, three questions were used to assess participants' confidence in their answers (7-point Likert scale), their subjective performance (numeric response), and their subjective judgment of what constitutes an acceptable amount of error for the task at hand (numeric response). Table 1 shows these questions.

- Subjective Experience: To understand the impact of our independent variables on how participants experienced each error type, we included questions from the NASA TLX cognitive load (CL) questionnaire [12], System Usability Scale (SUS) questionnaire [4], and a question asking about the realism of the gaze behavior. A 7-point Likert scale was used 
Table 1: The subjective Performance questionnaire.

\begin{tabular}{c|l}
\hline ID & Question \\
\hline SP1 & $\begin{array}{l}\text { How confident were you on the correctness of your } \\
\text { choices in this section of the experiment? }\end{array}$ \\
\hline SP2 & $\begin{array}{l}\text { What percentage of the targets do you think you identi- } \\
\text { fied correctly from 0\% to a 100\%? }\end{array}$ \\
\hline SP3 & $\begin{array}{l}\text { What do you think is an acceptable error margin for the } \\
\text { system, based on your assessment of your performance? }\end{array}$ \\
\hline
\end{tabular}

Table 2: The subjective Experience questionnaire.

\begin{tabular}{c|l}
\hline ID & Question \\
\hline CL1 & How mentally demanding was the task? \\
\hline CL2 & $\begin{array}{l}\text { How hard did you have to work to accomplish your } \\
\text { level of performance? }\end{array}$ \\
\hline CL3 & $\begin{array}{l}\text { How insecure, discouraged, irritated, stressed, or an- } \\
\text { noyed were you? }\end{array}$ \\
\hline SUS1 & I thought the system was easy to use. \\
\hline SE1 & I felt the gaze behavior of my partner was realistic. \\
\hline
\end{tabular}

Table 3: The adjusted Trust in Technology questionnaire.

\begin{tabular}{c|l}
\hline ID & Question \\
\hline TT1 & $\begin{array}{l}\text { The shared gaze system is a very reliable piece of soft- } \\
\text { ware. }\end{array}$ \\
\hline TT2 & $\begin{array}{l}\text { The shared gaze system has the features required for } \\
\text { my task. }\end{array}$ \\
\hline TT3 & $\begin{array}{l}\text { I am totally comfortable working with the shared gaze } \\
\text { system. }\end{array}$ \\
\hline TT4 & $\begin{array}{l}\text { I believe that most technologies are effective at what } \\
\text { they are designed to do. }\end{array}$ \\
\hline TT5 & $\begin{array}{l}\text { I usually trust a technology until it gives me a reason } \\
\text { not to trust it. }\end{array}$ \\
\hline
\end{tabular}

for all the questions in this questionnaire. Table 2 shows the questions used for this questionnaire.

- Trust in Technology: To assess participants' overall trust in technology and the shared gaze interface, we included several questions from Mcknight et al.'s Trust in Technology questionnaire [22]. A 7-point Likert scale was used for all items and they were adjusted to match our interface. This questionnaire, shown in Table 3, was presented to the participants after they completed all four blocks.

3.3.4 Hypotheses. Based on the literature (see Section 2) and a hypothesis-generating pilot study with five participants (different from our study population, who generally made conservative estimates on their own performance), we formalized the following hypotheses:

- H1: Participants' response time and error rate will increase as the error levels increase within each error type.

- H2: Participants' response time and error rate will increase as the target distance increases within each error type.
- H3: Based on the inherent nature of accuracy and latency errors that provide a constant spatial and temporal offset, compared to precision and dropout errors, participants will: a Give lower SP1 scores for the former error types and indicate less confidence in their answers,

b Give higher scores for CL1, CL2, CL3 for the former error types and indicate higher cognitive load,

c Give a lower SUS1 score for the former error types, assessing them as more difficult to use.

- H4: Participants' subjective estimate of the percentage of correctly identified targets answered through SP2 will be lower than their actual performance.

\section{RESULTS}

In this section we present the objective and subjective results for our experimental conditions. For the analysis of our results, we removed the data of one of our participants as it failed our sanity checks; we noticed that several responses were for targets that were located in completely opposite places compared to the actual target.

\subsection{Objective Measures}

We analyzed the results for the objective performance measures with repeated-measures ANOVAs and Tukey multiple comparisons with Bonferroni correction at the 5\% significance level. We confirmed the normality with Shapiro-Wilk tests at the $5 \%$ level and QQ plots. Degrees of freedom were corrected using GreenhouseGeisser estimates of sphericity when Mauchly's test indicated that the assumption of sphericity had been violated.

\subsubsection{Accuracy.}

Response Time. For accuracy, we found a significant main effect of error level, $F(3.21,61.09)=13.02, \boldsymbol{p}<\boldsymbol{0 . 0 0 1}, \eta_{p}^{2}=0.4$, no significant main effect of target distance, $F(2,38)=1.43, p=0.25$, $\eta_{p}^{2}=0.07$, and a significant interaction between the two factors, $F(4.98,94.71)=2.63, \boldsymbol{p}=\mathbf{0 . 0 2}, \eta_{p}^{2}=0.12$. Figure 3 (a) shows the aggregated response time for the simulated accuracy error levels at different target distances.

Error Rate. For accuracy, we found a significant main effect of error level, $F(2.99,56.84)=4.91, \boldsymbol{p}=\mathbf{0 . 0 0 4}, \eta_{p}^{2}=0.2$. We did not find a significant main effect of target distance, $F(2,38)=0.41, p=0.66$, $\eta_{p}^{2}=0.02$, suggesting that the tested target distances did not have a noticeable impact on participants' accuracy of responses. Figure 3(b) shows the aggregated error rate for the simulated accuracy error levels at different target distances.

\subsubsection{Precision.}

Response Time. For precision, we did not find a significant effect of error level, $F(2.79,53.141)=1.04, p=0.37, \eta_{p}^{2}=0.05$, and target distance, $F(1.61,30.73)=2.08, p=0.15, \eta_{p}^{2}=0.09$, on participants' response time, suggesting that the tested target distances and error levels did not noticeably add to the difficulty of the target identification task for this type of error. Figure 3(c) shows the aggregated response time for the simulated precision error levels at different target distances. 


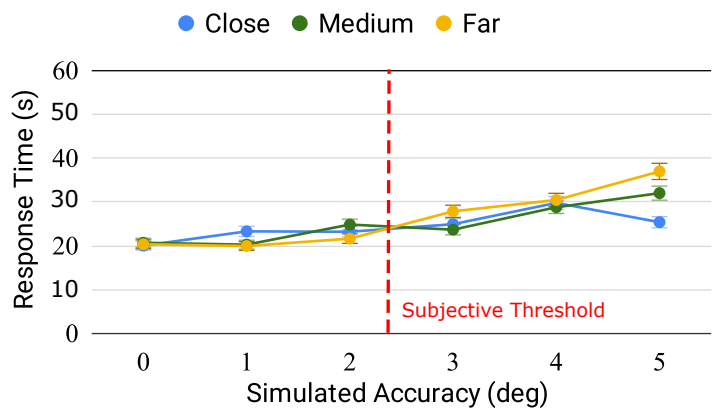

(a) Accuracy

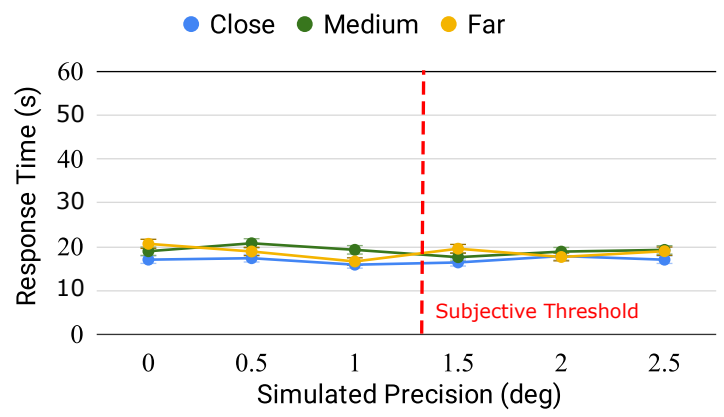

(c) Precision

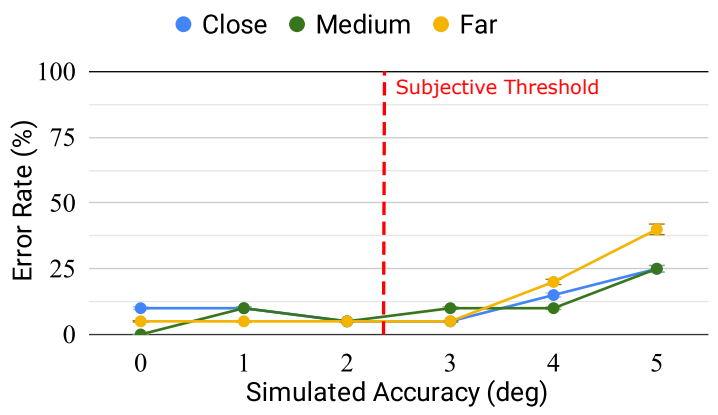

(b) Accuracy

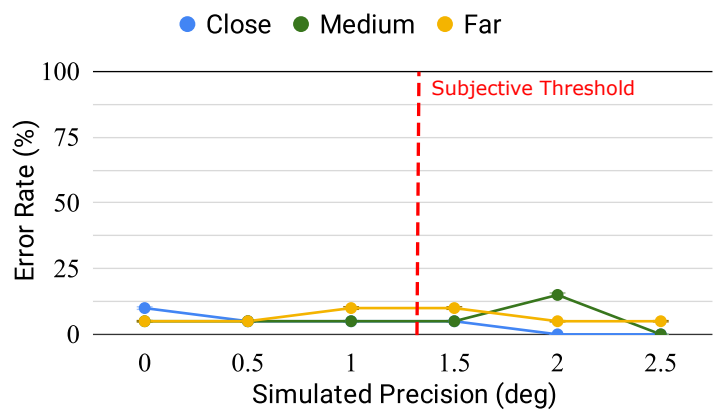

(d) Precision

Figure 3: Performance results related to eye tracking errors: The top row (a,b) shows results for accuracy and the bottom row (c,d) for precision. The left column (a,c) shows results for response time and the right column (b,d) for error rate.

Error Rate. For precision, we did not find a significant effect of error level, $F(5,95)=0.91, p=0.47, \eta_{p}^{2}=0.04$, and target distance, $F(2,38)=0.37, p=0.68, \eta_{p}^{2}=0.01$, on participants' error rate, again suggesting that the tested target distances and error levels did not noticeably add to the difficulty of the target identification task for this type of error. Figure 3(d) shows the aggregated error rate for the simulated precision error levels at different target distances.

\subsubsection{Latency.}

Response Time. For latency, we found a significant main effect of error level, $F(5,95)=16.12, \boldsymbol{p}<\mathbf{0 . 0 0 1}, \eta_{p}^{2}=0.45$. We did not find a significant main effect of target distance, $F(1.61,30.64)=1.27$, $p=0.27, \eta_{p}^{2}=0.06$, suggesting that the tested target distances did not noticeably add to the difficulty of the target identification task. Figure 4(a) shows the aggregated response time for the simulated latency error levels at different target distances.

Error Rate. For latency, we found a significant main effect of error level, $F(2.96,56.32)=14.23, \boldsymbol{p}<\boldsymbol{0 . 0 0 1}, \eta_{p}^{2}=0.42$. We also found a significant main effect of target distance, $F(2,38)=5.18$, $\boldsymbol{p}=\mathbf{0 . 0 1}, \eta_{p}^{2}=0.21$. Figure $4(\mathrm{~b})$ shows the aggregated error rate for the simulated latency error levels at different target distances.

\subsubsection{Dropout.}

Response Time. For dropout, we found a significant main effect of error level, $F(3.41,64.80)=26.26, \boldsymbol{p}<\mathbf{0 . 0 0 1}, \eta_{p}^{2}=0.58$, no significant main effect of target distance, $F(1.61,30.74)=0.61$, $p=0.51, \eta_{p}^{2}=0.03$, and a significant interaction between the two factors, $F(7.81,148.56)=2.68, \boldsymbol{p}=\mathbf{0 . 0 0 9}, \eta_{p}^{2}=0.12$. Figure $4(\mathrm{c})$ shows the aggregated response time for the simulated dropout error levels at different target distances.

Error Rate. For dropout, we did not find a significant main effect of error level, $F(2.59,49.28)=0.74, p=0.51, \eta_{p}^{2}=0.03$, or target distance, $F(1.39,26.55)=1.30, p=0.27, \eta_{p}^{2}=0.06$, on participants' error rate, which suggests that the tested target distances and error levels did not noticeably impact participants' responses. Figure 4(d) shows the aggregated error rate for the simulated dropout error levels at different target distances.

\subsection{Subjective Measures}

For the subjective questionnaire responses with an ordinal data type, we used non-parametric statistical tests to analyze the responses. We used Wilcoxon signed-ranks tests for the related samples.

4.2.1 Subjective Performance. We analyzed the results for the Performance questionnaire (Table 1) with respect to questions SP1, SP2, and SP3. See Table 4. 


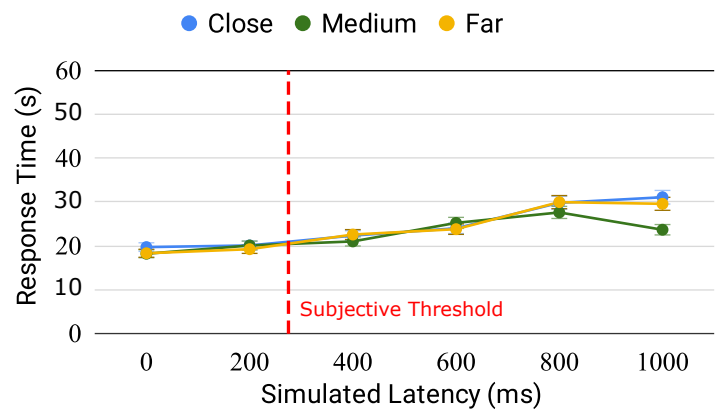

(a) Latency

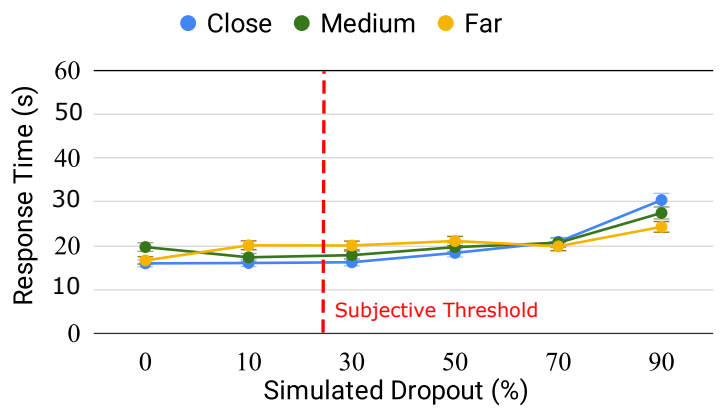

(c) Dropout

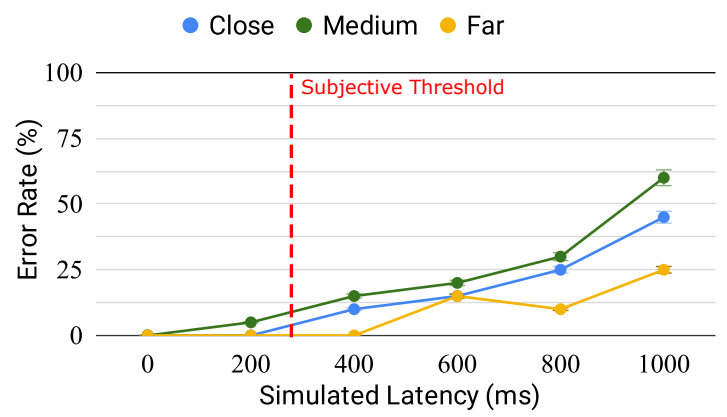

(b) Latency

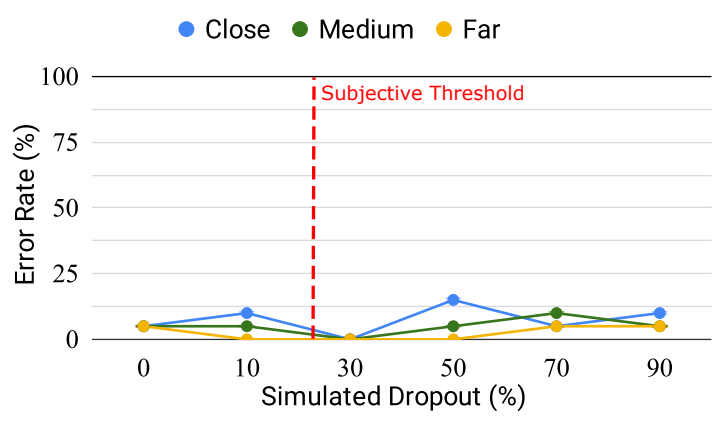

(d) Dropout

Figure 4: Performance results related to network errors: The top row (a,b) shows results for latency and the bottom row (c,d) for dropout. The left column (a,c) shows results for response time and the right column (b,d) for error rate.

For SP1, we did not find a significant difference among the error types when assessing participants' confidence in their responses, and the similar results for the different error types suggest that they did not have a noticeable impact on self-assessments of their performance.

However, for SP2, we found significant differences between participants' subjective assessment of performance and their actual performance for accuracy, $F(1,19)=8.07, \boldsymbol{p}=\mathbf{0 . 0 1}, \eta_{p}^{2}=0.29$, precision, $F(1,19)=20.05, \boldsymbol{p}<\mathbf{0 . 0 0 1}, \eta_{p}^{2}=0.51$, and dropout, $F(1,19)=29.16, \boldsymbol{p}<\boldsymbol{0 . 0 0 1}, \eta_{p}^{2}=0.6$. We observed a trend for latency, $F(1,19)=3.23, p=0.08, \eta_{p}^{2}=0.14$. These results are shown in Figure 5 and suggest that participants subjectively self-judged their performance as worse than what it actually was.

For SP3, we further looked at participants' subjective judgment of what they think constitutes the threshold for an acceptable amount of error. We identified a subjective accuracy threshold, $M=2.35 \mathrm{deg}, S D=1.37 \mathrm{deg}$, precision threshold, $M=1.36 \mathrm{deg}$, $S D=0.64 \mathrm{deg}$, latency threshold, $M=265.9 \mathrm{~ms}, S D=267.7 \mathrm{~ms}$, and dropout threshold, $M=23.35 \%, S D=17.49 \%$. We added these subjective thresholds as vertical red lines to the objective measures shown in Figures 3 and 4 . It is interesting to observe that these subjective thresholds seem to be in line with a drop in objective performance for accuracy and latency, while they do not seem to match changes in performance for precision and dropout.

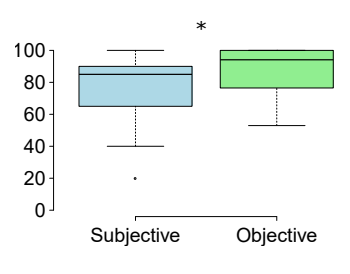

(a) Accuracy

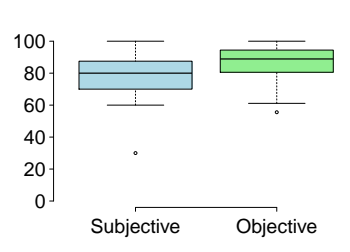

(c) Latency

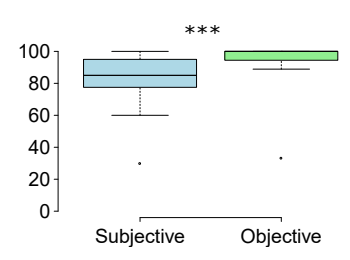

(b) Precision

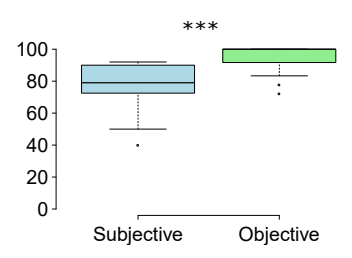

(d) Dropout
Figure 5: Comparison of participants' subjective estimates of their performance and their objective performance in terms of correctly identified targets during the experiment for (a) accuracy, (b) precision, (c) latency, and (d) dropout. Statistical significance: ${ }^{* * *}(\mathbf{p}<0.001),{ }^{* *}(\mathbf{p}<0.01),{ }^{*}(\mathbf{p}<0.05)$. 
Table 4: Subjective responses for the Performance and Experience questionnaires. We report the means (standard deviations) for the four error conditions.

\begin{tabular}{c|c|c|c|c}
\hline ID & Accuracy & Precision & Latency & Dropout \\
\hline SP1 & $5.30(1.38)$ & $5.55(1.57)$ & $5.10(1.37)$ & $5.00(1.29)$ \\
\hline SP2 & $77(20.93)$ & $83.40(16.94)$ & $78.00(16.17)$ & $77.10(14.43)$ \\
\hline SP3 & $2.35(1.37)$ & $1.36(0.64)$ & $265.9(267.7)$ & $23.35(17.49)$ \\
\hline CL1-3 & $3.78(1.34)$ & $3.00(1.27)$ & $4.01(1.25)$ & $3.60(1.05)$ \\
\hline SUS1 & $4.55(1.46)$ & $5.05(1.66)$ & $4.35(1.53)$ & $4.55(1.50)$ \\
\hline SE1 & $3.33(1.46)$ & $4.38(2.03)$ & $4.00(1.89)$ & $3.76(1.75)$ \\
\hline
\end{tabular}

4.2.2 Subjective Experience. We analyzed the results for the Experience questionnaire (Table 2) with respect to cognitive load questions CL1, CL2, and CL3, as well as ease of use question SUS1, and realism question SE1. See Table 4.

To measure the cognitive load related to each error type, we computed the mean value of CL1, CL2, and CL3. We found significant differences between precision and accuracy, $W=126, Z=3.01$, $\boldsymbol{p}=\boldsymbol{0 . 0 0 3}$, between precision and latency, $W=124, Z=2.9, \boldsymbol{p}=$ 0.004, and between precision and dropout, $W=162, Z=2.13, \boldsymbol{p}=$ $\mathbf{0 . 0 3}$, indicating that the levels of precision error were less mentally demanding. We did not find significant differences for the other comparisons.

For SUS1, we did not find significant differences among the different error types, with overall similar results indicated for ease of use.

For SE1, looking at realism of gaze behavior, we found significant differences between precision and accuracy, $W=13, Z=2.99, \boldsymbol{p}=$ 0.003, and a trend between accuracy and latency, $W=31, Z=1.68$, $p=0.09$.

4.2.3 Trust in Technology. The adapted Trust in Technology questionnaire (Table 3) was completed by participants at the end of the experiment. We analyzed the results with respect to questions TT1, TT2, and TT3 focused on participants' perception of the shared gaze interface and questions TT4 and TT5 measuring participants' general view of technology. We calculated the mean values of the questions in each group for TT1-3 $(M=4.78, S D=1.09)$ and TT4-5 $(M=4.50, S D=1.07)$.

We found a significant Pearson correlation between questions focusing on the shared gaze interface and technology, $r=0.701$, $\boldsymbol{p}=\boldsymbol{0 . 0 0 1}$. This suggests that participants who have a more trusting outlook towards technology rated the shared gaze interface as better even though 60 out of the 72 trials included some amount of error.

\subsection{Qualitative Feedback}

We conducted short interviews after the experiment to get a better understanding of our participants' impressions of the experience. Most of our participants indicated some level of discomfort associated with wearing the HoloLens for the duration of the experiment. Eleven of our participants reported experiencing slight or moderate amounts of eye strain. Only two of our participants reported experiencing slight amounts of headache or dizziness.

We asked our participants whether or not they observed differences in the gaze behavior of their simulated virtual partner within each block and between any two blocks to gauge how perceptible the simulated differences in error types were. Only three of our participants mentioned that the gaze behaviors within each block were similar and seemed to follow a consistent model. Apart from one participant who mentioned that the gaze behavior seemed similar comparing any two blocks, six participants noted that some blocks had similarities with each other. Interestingly, one of our participants mentioned the impact of the limited field of view of the HoloLens and that they tried to compensate for it by leaning slightly backwards while standing in place.

Some participants remarked on the fact that the gaze rays did not terminate at the body of the target human, which would provide useful depth cues for practical shared gaze environments. As discussed in Section 1, we expected this feedback, but such approaches would require accurate real-time information about dynamic scene geometry, which is highly challenging for practical applications, and not considered in the scope of this paper.

Most participants further indicated that they made judgments based on a visual comparison between the movement patterns of the target humans and those of the gaze ray, and that it helped them in completing the task. It would be more challenging to identify a stationary target among a group of stationary objects. That is, unless one's simulated partner is moving as well, which could provide similar cues as in this study, and would be interesting for future research.

\section{DISCUSSION}

In this experiment, we observed different effects on participants' performance for error types when error level and target distance were varied. We also noticed the relatively high performance of many of our participants even though the subjective estimates of their performance were lower. Last but not least, we identified subjective thresholds for tolerable error levels. In this section, we discuss our findings and their implications for practitioners.

\subsection{Effects of Error Type on Performance and Subjective Response}

To answer our initial research question RQ1, based on the differences in the nature of each error, we predicted that accuracy and latency errors can impact participants' performance more negatively. Indeed, this can be observed in Figure 5. In the case of our task, the temporal and spatial offset introduced through accuracy and latency errors led to more misinterpretations than precision and dropout errors. This offset posed a bigger potential for the gaze ray to be on the wrong human for a longer duration of time, causing temporary misinterpretations similar to Holmqvist et al.'s example for accuracy errors [13]. This required participants to rely more on the movement patterns of the human targets and spend more time on target identification.

We observed that our participants subjectively assessed their performance to be worse than their actual performance, supporting our Hypothesis $\mathbf{H 4}$ and suggesting a response to part of our research question RQ3, which is similar to Waltemate et al.'s findings on how participants had a tendency to rather perceive themselves as the cause of error in a visual movement task rather than an introduced system error [31]. We also observed a lower score for 
cognitive load for precision compared to other error types, partly supporting our Hypothesis H3b. Surprisingly, we did not find a difference for SP1 (i.e., confidence in answers) and SUS1 (i.e., ease of use), thus not supporting our Hypotheses H3a and H3c. We think that the novelty of the interaction, some perceived similarity among blocks, and participants' generally lower self-assessment of performance might have caused them to give similar confidence scores for the different error types, although it is important to note that for all error types the mean confidence was higher than 5 on a scale of 1 to 7 .

\subsection{Effects of Error Level on Performance and Subjective Error Estimation}

We found multiple effects indicating a relative increase in response time and a decrease in error rate for simulated error levels for different error types, partly confirming our Hypothesis H1. To answer our second research question RQ2, we collected subjective estimates of what participants indicated as tolerable thresholds for error levels. Their responses indicate that for our task, thresholds of $2.35 \mathrm{deg}$ for accuracy, $1.36 \mathrm{deg}$ for precision, $265.9 \mathrm{~ms}$ for latency, and $23.35 \%$ for dropout were acceptable.

We compared these subjective thresholds to the objective performance for accuracy and latency and found that they indeed seem to be indicating an objective drop in performance, which is supported by our statistical analysis. For less demanding situations, we would go as far as to say that thresholds of $3.5 \mathrm{deg}$ for accuracy and $600 \mathrm{~ms}$ for latency are acceptable before the performance drops more drastically. Practitioners may select an appropriate level of performance based on their constraints with respect to the temporal demands and severity of errors of the task at hand, and invest in corresponding eye trackers and network solutions.

In contrast, the subjective thresholds do not seem to match noticeable changes in performance for precision and dropout. For dropout, we observed most of the significant differences in performance around the highest error value in the tested range (i.e., $90 \%$ chance of frame drops). We did not observe a change in performance for precision throughout the tested range, suggesting that our tested values were not large enough to cause any disruptions in our participants' performance. We would like to point out that we chose these ranges based on reported error levels in the literature and by eye tracker manufacturers (Section 3.2), and that it is encouraging for shared gaze applications if these levels are already tolerable.

\subsection{Effects of Target Distance on Performance}

In contrast to what we had hypothesized in $\mathbf{H} 2$, there were only a few instances of error types and error levels where we observed a significant decrease in performance for some target distances, and this was not always for the farthest targets. Although there were instances where we saw a significant decrease in performance as the targets were further back such as for accuracy errors, we also observed the opposite effect for some latency and dropout error levels where the response time and error rate was higher for targets at the close and medium distances. We think that this can partly be explained by the limitations in the field of view of the HoloLens HMD used for the experiment. It was more difficult for participants to see the AR gaze ray and the human targets at a close distance since they could easily move out of the augmented field of view.

\subsection{Limitations}

To fully control for behavioral factors among participants and the trials, in this experiment we chose to use a simulated virtual human partner. Although we did not measure for social presence and copresence among collaborators, we understand that the social impact of the partner could have been different if a real person was chosen instead or if the virtual human partner initiated a conversation with the participants during the experiment. As a separate experiment, it would be interesting to investigate how the different partners (i.e., real or virtual) and level of interaction with that partner might influence the overall collaborative experience although we expect that the perception of gaze should remain the same for the different partners.

Separately, we chose the same viewing direction for both the participants and the simulated partner, who were standing at a relatively close distance to each other (i.e., one meter). It is important to note that the participants' position relative to the virtual human collaborator, as well as the participants' distances to the targets likely have a significant impact on participants' performance and their overall experience. While not investigated here, future work should evaluate the role that such factors play in remote collaboration experiences.

\section{CONCLUSION AND FUTURE WORK}

In this paper, we investigated the effects of error type, error level, and target distance in an AR shared gaze interface on participants' objective performance and subjective responses through a controlled human-subject study. We designed an experimental scenario inspired by a practical use case of two police officers scanning a crowd of people for a potential threat. In our study, participants were asked to collaborate with a simulated virtual partner and leverage their AR gaze ray to identify a target human among a crowd. We introduced different errors that could impact the data quality presented to the participants either caused by an eye tracker or the network used and measured participants' performance through their response time and error rate in identifying the targets and assessed their subjective experience.

We identified thresholds for acceptable amounts of error. Our findings suggest that eye tracker accuracy and network latency experienced in current-state shared gaze setups have a noticeable effect on users' performance. In contrast, the tested common ranges of errors for precision and lag were largely acceptable, indicating that these are not a major performance concern for practitioners. We further observed that the field of view of current-state AR HMDs can affect participants' performance with regards to different target distances, and we plan to explore this factor in future work. We also plan to investigate the impact of other visualization techniques, with or without available dynamic scene geometry information, for the gaze cues and how they compare to the gaze ray used in this experiment. 


\section{ACKNOWLEDGMENTS}

This material includes work supported in part by the National Science Foundation under Award Number 1564065 (Dr. Ephraim P. Glinert, IIS) and Collaborative Award Numbers 1800961, 1800947, and 1800922 (Dr. Tonya Smith-Jackson, IIS) to the University of Central Florida, University of Florida, and Stanford University respectively; the Office of Naval Research under Award Number N0001417-1-2927 (Dr. Peter Squire, Code 34); and the AdventHealth Endowed Chair in Healthcare Simulation (Prof. Welch). Any opinions, findings, and conclusions or recommendations expressed in this material are those of the author(s) and do not necessarily reflect the views of the supporting institutions.

\section{REFERENCES}

[1] Michael Barz, Andreas Bulling, and Florian Daiber. 2015. Computational modelling and prediction of gaze estimation error for head-mounted eye trackers. DFKI ResearchReports 1, 1 (2015), 1-10.

[2] Martin Bauer, Gerd Kortuem, and Zary Segall. 1999. "Where are you pointing at?" A study of remote collaboration in a wearable videoconference system. In Digest of Papers. Third International Symposium on Wearable Computers. IEEE, 151-158.

[3] Susan E Brennan, Xin Chen, Christopher A Dickinson, Mark B Neider, and Gregory J Zelinsky. 2008. Coordinating cognition: The costs and benefits of shared gaze during collaborative search. Cognition 106, 3 (2008), 1465-1477.

[4] John Brooke et al. 1996. SUS-A quick and dirty usability scale. Usability evaluation in industry 189, 194 (1996), 4-7.

[5] Juan J Cerrolaza, Arantxa Villanueva, Maria Villanueva, and Rafael Cabeza. 2012 Error characterization and compensation in eye tracking systems. In Proceedings of the Symposium on Eye Tracking Research and Applications. ACM, 205-208.

[6] Jan Drewes, Guillaume S Masson, and Anna Montagnini. 2012. Shifts in reported gaze position due to changes in pupil size: Ground truth and compensation. In Proceedings of the Symposium on Eye Tracking Research and Applications. ACM 209-212.

[7] Stephen R Ellis, Francois Breant, B Manges, Richard Jacoby, and Bernard D Adelstein. 1997. Factors influencing operator interaction with virtual objects viewed via head-mounted see-through displays: viewing conditions and rendering latency. In Proceedings of IEEE Annual International Symposium on Virtual Reality. IEEE, 138-145.

[8] Anna Maria Feit, Shane Williams, Arturo Toledo, Ann Paradiso, Harish Kulkarni, Shaun Kane, and Meredith Ringel Morris. 2017. Toward everyday gaze input Accuracy and precision of eye tracking and implications for design. In Proceedings of the Chi Conference on Human Factors in Computing Systems. ACM, 1118-1130.

[9] Kay Fitzpatrick, Marcus A Brewer, and Shawn Turner. 2006. Another look at pedestrian walking speed. Transportation Research Record 1982, 1 (2006), 21-29.

[10] Erik Geelhoed, Aaron Parker, Damien J. Williams, and Martin Groen. 2009. Effects of latency on telepresence. Technical Report HPL-2009-120. HP Laboratories.

[11] Kunal Gupta, Gun A Lee, and Mark Billinghurst. 2016. Do you see what I see? The effect of gaze tracking on task space remote collaboration. IEEE Transactions on Visualization and Computer Graphics 22, 11 (2016), 2413-2422.

[12] Sandra G Hart and Lowell E Staveland. 1988. Development of NASA-TLX (Task Load Index): Results of empirical and theoretical research. In Advances in Psychology. Vol. 52. Elsevier, 139-183.

[13] Kenneth Holmqvist, Marcus Nyström, and Fiona Mulvey. 2012. Eye tracker data quality: what it is and how to measure it. In Proceedings of the Symposium on Eye Tracking Research and Applications. ACM, 45-52.

[14] Anthony J Hornof and Tim Halverson. 2002. Cleaning up systematic error in eye-tracking data by using required fixation locations. Behavior Research Methods, Instruments, \& Computers 34, 4 (2002), 592-604.
[15] Sophie Jörg, Aline Normoyle, and Alla Safonova. 2012. How responsiveness affects players' perception in digital games. In Proceedings of the ACM Symposium on Applied Perception. ACM, 33-38.

[16] Kangsoo Kim, Mark Billinghurst, Gerd Bruder, Henry Been-Lirn Duh, and Gregory F. Welch. 2018. Revisiting Trends in Augmented Reality Research: A Review of the 2nd Decade of ISMAR (2008-2017). IEEE Transactions on Visualization and Computer Graphics (TVCG) 24, 11 (2018), 2947-2962.

[17] Kangsoo Kim, Arjun Nagendran, Jeremy Bailenson, and Greg Welch. 2015. Expectancy Violations Related to a Virtual Human's Joint Gaze Behavior in RealVirtual Human Interactions. In Proceedings of International Conference on Computer Animation and Social Agents. 5-8.

[18] Kiyoshi Kiyokawa, Haruo Takemura, and Naokazu Yokoya. 1999. A collaboration support technique by integrating a shared virtual reality and a shared augmented reality. In IEEE Proceedings of the International Conference on Systems, Man, and Cybernetics (Cat. No. 99CH37028), Vol. 6. IEEE, 48-53.

[19] Alexandros Koilias, Christos Mousas, and Christos-Nikolaos Anagnostopoulos. 2019. The Effects of Motion Artifacts on Self-Avatar Agency. In Informatics, Vol. 6. Multidisciplinary Digital Publishing Institute, 18.

[20] Stephen RH Langton, Roger J Watt, and Vicki Bruce. 2000. Do the eyes have it? Cues to the direction of social attention. Trends in Cognitive Sciences 4, 2 (2000), 50-59.

[21] Cha Lee, Scott Bonebrake, Doug A Bowman, and Tobias Höllerer. 2010. The role of latency in the validity of AR simulation. In IEEE Virtual Reality Conference (VR). 11-18.

[22] D Harrison Mcknight, Michelle Carter, Jason Bennett Thatcher, and Paul F. Clay. 2011. Trust in a specific technology: An investigation of its components and measures. ACM Transactions on Management Information Systems 2, 2 (2011), 12.

[23] Marcus Nyström, Richard Andersson, Kenneth Holmqvist, and Joost Van De Weijer. 2013. The influence of calibration method and eye physiology on eyetracking data quality. Behavior Research Methods 45, 1 (2013), 272-288.

[24] Kristien Ooms, Lien Dupont, Lieselot Lapon, and Stanislav Popelka. 2015. Accuracy and precision of fixation locations recorded with the low-cost Eye Tribe tracker in different experimental setups. Fournal of Eye Movement Research 8, 1 (2015), 1-20.

[25] Andriy Pavlovych and Wolfgang Stuerzlinger. 2011. Target following performance in the presence of latency, jitter, and signal dropouts. In Proceedings of Graphics Interface. Canadian Human-Computer Communications Society, 33-40.

[26] Thammathip Piumsomboon, Arindam Day, Barrett Ens, Youngho Lee, Gun Lee, and Mark Billinghurst. 2017. Exploring enhancements for remote mixed reality collaboration. In ACM SIGGRAPH Asia Mobile Graphics \& Interactive Applications. 16.

[27] Thammathip Piumsomboon, Youngho Lee, Gun A Lee, Arindam Dey, and Mark Billinghurst. 2017. Empathic mixed reality: Sharing what you feel and interacting with what you see. In International Symposium on Ubiquitous Virtual Reality. IEEE, 38-41.

[28] Eric Ragan, Curtis Wilkes, Doug A Bowman, and Tobias Hollerer. 2009. Simulation of augmented reality systems in purely virtual environments. In IEEE Virtual Reality Conference. 287-288.

[29] Katrin Schoenenberg. 2016. The Quality of Mediated-Conversations under Transmission Delay. Ph.D. Dissertation. TU Berlin.

[30] Nicholas Toothman and Michael Neff. 2019. The Impact of Avatar Tracking Errors on User Experience in VR. In Proceedings of IEEE Virtual Reality (VR). 1-11.

[31] Thomas Waltemate, Irene Senna, Felix Hülsmann, Marieke Rohde, Stefan Kopp, Marc Ernst, and Mario Botsch. 2016. The impact of latency on perceptual judgments and motor performance in closed-loop interaction in virtual reality. In Proceedings of the ACM Conference on Virtual Reality Software and Technology. 27-35.

[32] Gregory Welch, Gerd Bruder, Peter Squire, and Ryan Schubert. 2019. Anticipating Widespread Augmented Reality: Insights from the 2018 AR Visioning Workshop. Technical Report. University of Central Florida and Office of Naval Research.

[33] Yanxia Zhang, Ken Pfeuffer, Ming Ki Chong, Jason Alexander, Andreas Bulling, and Hans Gellersen. 2017. Look together: using gaze for assisting co-located collaborative search. Personal and Ubiquitous Computing 21, 1 (2017), 173-186. 